Faculty of Engineering, Imperial College London, SW7 2AZ, United Kingdom, 'School of Life and Health Sciences, Aston University, Aston Triangle, Birmingham B4 7ET, United Kingdom n.chayen@imperial.ac.uk

A method for identifying protein crystals based on Attenuated Total Reflection Fluorescence Transmission Infrared Spectroscopy (ATR-FT-IR) is presented [1]. This approach uses imaging, through a combination of chemical specificity and the visualizing capability and is complementary to detection of crystals using Ultraviolet (UV) light. ATR-FT-IR imaging was successfully applied to study the crystallization of several proteins simultaneously from six different solutions, using microbatch under oil. The technique is fast as it studies protein crystallization in situ and provides an opportunity to examine many different samples under a range of conditions. Studies applying this technique with other proteins are underway.

[1] Chan, K.L.A., Govada, L., Bill, R.M., Chayen, N.E. \& Kazarian, S.G. Analytical Chemistry ,2009,81, 3769-3775

Keywords: protein crystallization-1, imaging-2, atr-ft-ir-3

\title{
FA5-MS38-P14
}

\section{Experimental Minstrel HT UV: Automated Imaging of Protein Drops with UV Fluorescence. Max} Petersen, Jian Xu, Michael Willis, Rigaku Automation, Inc., USA

E-mail: Max.Petersen@Rigaku.com

The Minstrel HT UV'M is a fully automated imager specifically designed for high-throughput ultraviolet and visible crystal imaging and monitoring of protein crystal growth. We have focused our research and development efforts on combing knowledge and skill of experts in optics, photochemistry, illumination, and automation to develop a custom solution that provides the highest sensitivity, the highest optical resolution, and the least photo damage to a protein sample. The result of this effort provides a substantial leap forward in imaging technology in both UV and visible color spectrum. The optimal balance of high resolution and depth of field for the crystallographic application allows for imaging of hanging drop, sitting drop, and microbatch experiments for all UV suitable plates. When combined with Rigaku's Gallery' 700 incubators, the Minstrel HT UV provides around the clock unattended incubation at user defined temperature settings and inspection schedules. Image analysis and the associated data management are handled by a software environment that not only allows for scoring of imaging results, but also reporting and management of all relevant crystallization information. Validation studies for various representative protein crystallization experiments show how the Minstrel HT UV imaging system is able to cope with complex drops, providing researchers with a significant advantage in the detection of protein crystals.

Keywords: protein crystallization, automation, microscopes 